\title{
INTRANEURONAL TRANSPORT: ROLE OF CALCIUM
}

\author{
By Zafar Iqbal*
}

DOI: http://dx.doi.org/10.5915/15-1-12380

Neuron, the structural unit of nervous tissue is a highly specialized cell comprising of perikaryon (cell body, soma) and its processes, the dendrites and the axon (axis cylinder, nerve fiber). The neuronal cells vary considerably in shape and size in different parts of the body. Granule cells from the cerebellum are $5 \mathrm{u}$ in diameter, large motor cells of the anterior horn of the spinal cord are up to $120 \mathrm{u}$ in diameter. The axon, at times extending over distances thousands of times greater than the perikaryon, does not have the capability to synthesize proteins and other cellular components required for its maintenance. Thus, it requires a continual supply of materials synthesized in the perikaryon by a process referred to as axoplasmic, axonal or neuroplasmic transport or flow (Fig. 1). This intracellular transport of a wide range of components serves to replace the structural components consumed in metabolism and provides materials such as neurotransmitters, neurotrophic substances and modulators required for synaptic function. This article describes some of the essential features of the transport and the role of $\mathrm{Ca}^{2}+$ in this process.

\section{Study of Transport}

There are several procedures employed in the study of the transport in neuronal cell. One method of studying the transport of materials in the nerve is to ligate the nerve fiber and analyze the material accumulated proximal to the ligation. Another method is to trap the transported material in nerve by 'cold block'. The exposure of nerves to cold temperature $\left(0-4^{\circ} \mathrm{C}\right)$ causes a block in the transport resulting in the accumulation of transported components (at the proximal end of the cold exposure areas) which are then analyzed.

The technique most commonly employed makes use of radioactive isotopes: a labelled precursor is injected into the ganglion, an area rich in cell bodies. The precursor is incorporated into macromolecular components in the cell soma and, with time, these components move out of their site of synthesis into the axon. The transported material is then detected by the determination of radioactivity in different nerve segments at various time intervals after the injection of the precursors. This procedure has been employed in our lab for over a

\footnotetext{
- Departments of Physiology and Biochemistry and Medical Biophysics, Indiana University School of Medicine, Indianapolis, IN
}

decade and the studies conducted with sciatic nerves of cat, rat, and monkeys have established the rate of fast transport in mammalian nerve as $410 \pm$ $10 \mathrm{~mm} /$ day or $17 \mathrm{~mm} / \mathrm{h}$ (Kidwai and Ochs, 1969; Ochs, 1972, 1974; Iqbal and Ochs, 1978a). The details of the technique are given in Fig. 2.

\section{Characteristics of Transport}

The transport of various substances has been found to be independent of the size of the nerve fibers, as well as the age of the mammalian species studied, however, variations in the rate of transport during the development of animals have been reported. The rate of transport in the myelinated and non-myelinated fibers appears to be the same.

The transport is an energy-dependent process. As such, any interruptions in the energy-related reactions block the transport (Sabri and Ochs, 1972; Ochs, 1974b).

The transport process has been observed to be effective only in the presence of microtubular structure in the cells. Antitumour agents which cause disassembly of the microtubules in nerve fibers block the axoplasmic transport (McClure, 1972; Chan et al, 1978). Our studies with radioactive labeled vinca agents have indicated that these antitumor agents are taken up by the nerve fibers, thus causing a block in the transport (Iqbal, 1979a, $1980 c, d$; Iqbal and Ochs, 1980). The accumulation of the cations inside the nerve fiber (for example, by exposing the desheathed cat sciatic nerve, allowing it to take up calcium from the medicine) also disassembles the microtubules and blocks the transport.

The axoplasmic transport of various substances has recently been found to be dependent on calcium. (Ochs, Worth and Chan, 1977; Ochs et al, 1978; Hammerschlag et al, 1977; Chan et al, 1980).

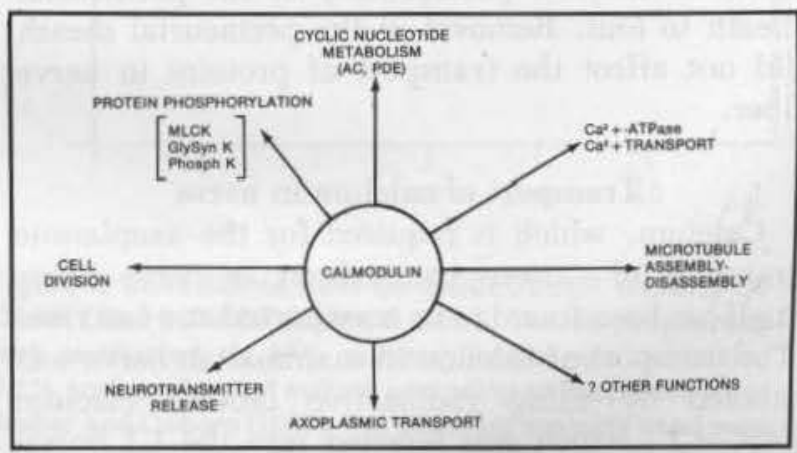

Figure 1. AXOPLASMIC TRANSPORT. 


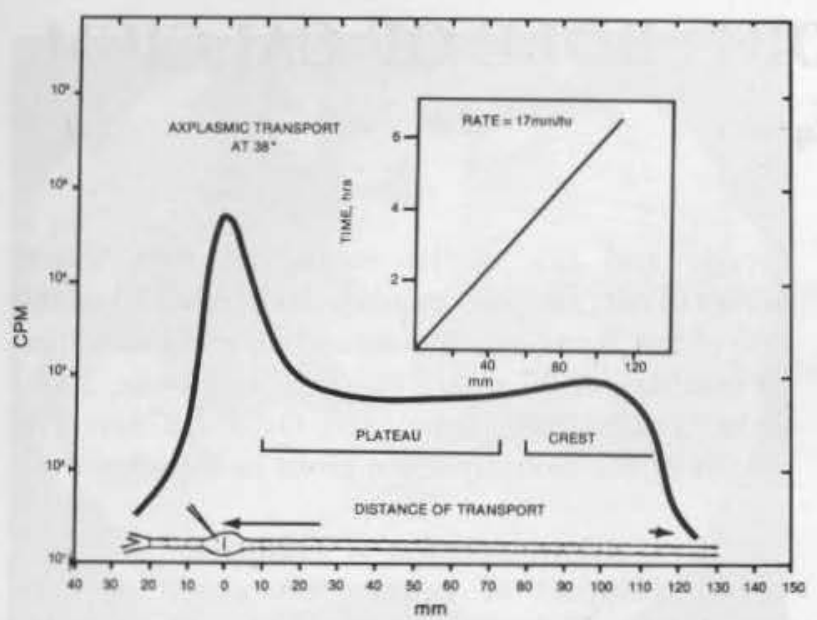

Figure 2. TECHNIQUE OF STUDY OF AXOPLASMIC TRANSPORT. Cats are anesthetized with barbital and laminectomy is performed to expose the L.7 dorsal root ganglion. A micropipette containing radioactive material ('H) leucine or $\left.{ }^{45} \mathrm{Ca}^{2}+\right)$ is inserted into the ganglion and about 10.151 of istope is injected into the ganglion with the help of a micromanipulator. After the injection, the ganglion is covered with a cloth wet with Kreb's Ringer solution and the temperature is maintained at $38^{\circ} \mathrm{C}$. The precursor is incorporated into the proteins and polypeptides. The transport of these labelled substances is determined by counting the radioactivity in the nerve segments. In this particular case the sciatic nerve was removed from the animal $7 \mathrm{~h}$ after the injection of the isotope into the ganglion and sliced into $5 \mathrm{~mm}$ segments. The ordinate in the graph shows the radioactivity (counts per minute, c.p.m.) in log scale plotted against the length of the nerve $(\mathrm{mm})$. The ganglion area, which is the site of injection and has a high level of activity, is taken as zero distance. With time, the labelled substances move down the nerve fibers and the fastest moving activity provides a 'crest' of activity. The rate of transport is determined by dividing the distance of the crest activity from the ganglion by time (insert). This gives a rate of $17 \mathrm{mmm} / \mathrm{h}$ at $38^{\circ} \mathrm{C}$.

Using a desheathed sciatic nerve preparation from cat in vitro, Chan, et al (1980) have shown that the removal of $\mathrm{Ca}^{2}+$ from the incubation medium blocks axoplasmic transport, this effect being reversed by adding $\mathrm{Ca}^{2}+$ to the medium. Earlier observations traced the failure of demonstration of the requirement of calcium for the transport in nerve to the low permeability of the perineurial sheath to ions. Removal of the perineurial sheath did not affect the transport of proteins in nerve fiber.

\section{Transport of calcium in nerve}

Calcium, which is required for the axoplasmic transport of various components in nerve fibers itself has been found to be transported at a fast rate. The transport of calcium in mammalian nerve was studied by using radioactive labeled calcium $\left(45 \mathrm{Ca}^{2+}\right)$ which was injected into the L7 dorsal root ganglion as described earlier (Iqbal and Ochs, 1978a). Cats were anesthetized with barbital and laminectomy was performed to expose the dorsal root ganglion. A micropipette containing radioactive material, $45 \mathrm{Ca}$, was inserted into the ganglion and about $2-5 \mathrm{ul}(5 \mathrm{mc} / \mathrm{ml})$ of isotope was injected into the ganglion with the help of a micromanipulator. The injection process was observed under a microscope. After the injection, the ganglion was covered with a cloth wet with Kreb's Ringer solution and the temperature was maintained as $38^{\circ} \mathrm{C}$. The transport of labeled substances was determined by counting the radioactivity in the nerve segments at various times after the injection of the isotope into the ganglion. The nerve was cleaned free of all adventitious material, washed with Kreb's Ringer solution and sliced into 3 or $5 \mathrm{~mm}$ segments and these nerve segments were each digested with tissue solubulizer, Soluene, and counted in a scintillation counter after adding $10 \mathrm{ml}$ of scintillation fluid consisting of PPO (2,5-diphenylazol) and POPOP (1,4-bis-2,5 phenylzol) Benzene in toluene. With time the labeled substances moved down the nerve fibers and the fastest moving activity was taken as a crest of the activity. The rate of the transport of $\mathrm{Ca}$ was determined by dividing the distance of crest of the activity from the ganglion by time. This gave a transport rate of $17 \mathrm{~mm} / \mathrm{h}$ for $\mathrm{Ca}^{2}+$ at $38^{\circ} \mathrm{C}$, a rate similar to other substances in the nerve.

To study the components which are transporting $45 \mathrm{Ca}^{2}+$ in the nerves, portions of the nerve containing the crest and the plateau region were taken 5 to 7 hours after the L7 dorsal root ganglion injection with the isotope. The nerve portions containing fast transported radioactivity and a segment distal to the fast transported region serving as control were cut into small pieces and homogenized in 0.32 $\mathrm{M}$ sucrose solution with a Polytron homogenizer at a speed of 4,000 rpm for two minutes in cold and subjected to subcellular fractionation by the conventional differential centrifugation technique. About $70 \%$ of the $45 \mathrm{Ca}$ was found to be associated with the mitochondrial and microsomal fractions, whereas $16-20 \%$ was located in the soluble fraction of the nerve (Igbal and Ochs, 1978). The soluble proteins were further characterized by gel filtration chromatography on Sephadex G-100 (40-100 u) equilibrated with a $10 \mathrm{mM}$ phosphate buffer at $\mathrm{pH}$ 7.2. Eight $\mathrm{ml}$ fractions of the eluate were collected using an LKB-fraction collector while the protein in the eluates was monitored by their $280 \mathrm{~nm}$ absorption using LKB UV cord-II. The soluble proteins fractionate into high (peak I) and low (peak II) molecular weight components when chromatographed on Sephadex G-100 column (Fig. $3)$. The specific activity (CPM/mg protein) in the 


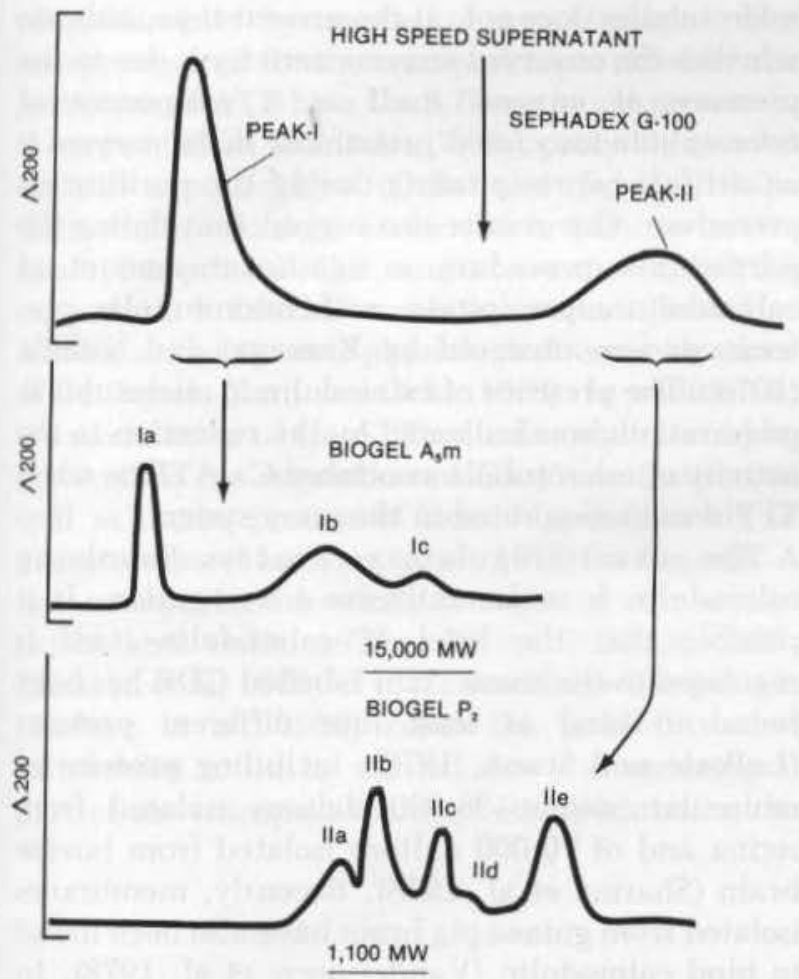

Figure 3. SCHEME FOR CHARACTERIZATION OF CA $^{2}+-$ ASSOCIATED COMPONENTS FAST TRANSPORTED IN NERVE. The soluble proteins containing fast transported components obtained after high speed centrifugation were fractionated on a column packed with Sephadex G-100. The column was equilibrated and eluted with $10 \mathrm{mM}$ phosphate buffer at ph 7.2 using an LK3-automatic fraction collector. Aliquots from each fraction were analyzed for protein and radioactivity as described in the text. Bound ${ }^{45} \mathrm{Ca}$. radioactivity eluted with peak I and peak II containing high and low molecular weight proteins. Further fractionation of peak I on BioGel A5m column resulted in the identification of a calcium-binding protein of about 15,000 dalton eluting with peak Ic. Among the low molecular species, bound $45 \mathrm{Ca}$ was found to be associated with a polypeptide of 1,100 dalton eluting with peak IIb from BioGel P2 column. Using $3 \mathrm{H}$-labeled leucine both Ic and IIb polypeptides were found to be transported at a fast rate in cat sciatic nerve.

peak I isolated from the nerve pieces containing the transported material was found to be about 10 times higher than that in control nerves. The components eluting in peak I from Sephadex G-100 appear to be an aggregate of three different protein groups. When peak I material was further fractionated on a column packed with BioGel A5m (Bio Rad Co.) three subpeaks Ia, Ib and Ic were obtained. Most of the bound $45 \mathrm{Ca}$ was found to be eluted with the subpeak Ic which was low in protein concentration. The bound $45 \mathrm{Ca}$ activity found in the control portions of the nerve was small and accounted for only approximately $1 / 10-1 / 20$ th of the bound activity carried down by fast axoplasmic transport in the nerve.

The protein, having a calcium-binding property, and hence referred to as calcium-binding protein (CaBP), was found to be transported at a fast rate in the nerve (Iqbal, 1978; Iqbal and Ochs, 1978a-c). When $(3 \mathrm{H})$-leucine was injected into the ganglion and the nerve segment corresponding to the fast transported crest of the activity was compared with the plateau of the activity, the specific activity of peak Ic from the crest region was found to be 2 to 3 -fold greater as compared to that in the plateau region.

Properties of CaBP: Similarity to calmodulin

$\mathrm{CaBP}$ is an acidic protein (pI, 3.9). It has strong affinity for $\mathrm{Ca}$ and elutes as a big molecular size moiety from the gel filtration media in the presence of $\mathrm{Ca}$. Removal of $\mathrm{Ca}$ leads to its elution as a 15,000 daltons moiety from the column. Purified $\mathrm{CaBP}$ has a molecular weight of $15,000-16,000$ daltons, as determined by its relative electrophoretic mobility on acrylamide gels containing SDS (Fig. 4). The protein has a wide distribution and the protein

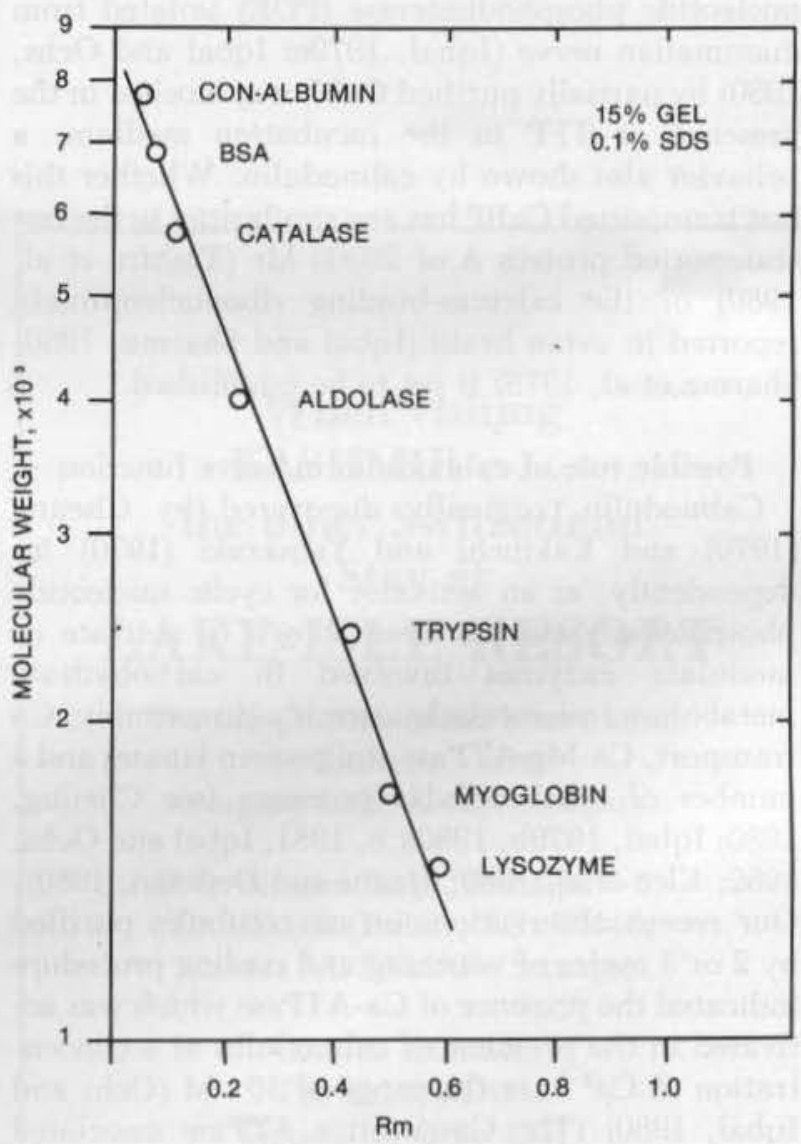

Figure 4. DETERMINATION OF MOLECULAR WEIGHT OF CABP ON POLYACRYLAMIDE GELS. The electrophoresis was conducted on $15 \%$ polyacrylamide gels containing $0.1 \%$ sodium dodecyl sulfate according to the procedure of Weber and Osborn (1969). The standard proteins used were conalbumin, BSA (bovine serum albumin, catalase, aldolase, trypsin, myoglobin, and lysozme. 
isolated from brain, spinal cord and nerve appears to be the same (Iqbal, 1979b). It can be synthesized at a rapid rate and transported to synapses (Iqbal and Ochs, 1978a-c).

The protein isolated from the nerve shows remarkable similarities to calmodulin. Like nerve $\mathrm{CaBP}$, calmodulin or calcium-dependent regulator protein (CDR) also has the property to bind to a number of proteins in the presence of $\mathrm{Ca}$ as shown by its elution profile from gel filtration columns. Calmodulin prepared from dog sciatic nerve and brain and the fast transported CaBP show identical electrophoretic mobilities on acrylamide gels containing sodium dodecyle sulfate and on isoelectrofocusing gels, indicating that these proteins are similar (Iqbal, 1979c,d, 1980d, 1981a,b; Iqbal and Ochs, 1979a,b, 1980a,e, 1982). Additional confirmation for the similarities of the protein with calmodulin came from the use of trifluoperazine (TFP), an agent which binds specifically to calmodulin in the presence of $\mathrm{Ca}$ (Levin and Weiss, 1978). The stimulation of partially purified cyclic nucleotide phosphodiesterase (PDE) isolated from mammalian nerve (Iqbal, 1979e; Iqbal and Ochs, 1980 ) by partially purified CaBP was blocked in the presence of TFP in the incubation medium, a behavior also shown by calmodulin. Whether this fast transported $\mathrm{CaBP}$ has any similarities to the fast transported protein A of $26000 \mathrm{Mr}$ (Tashiro et al, 1980 ) or the calcium-binding ribonucleoprotein reported in avian brain (Iqbal and Sharma, 1980; Sharma et al, 1975) is yet to be established.

\section{Possible role of calmodulin in nerve function}

Calmodulin, orginally discovered by Cheung (1970) and Kakiuchi and Yamazaki (1970) independently, as an activator for cyclic nucleotide phosphodiesterase has been shown to activate or modulate enzymes involved in carbohydrate metabolism, microtubule assembly-disassembly, Ca transport, $\mathrm{Ca}-\mathrm{Mg}$-ATPase and protein kinases and a number of other cellular processes (see Cheung, 1980; Iqbal, 1979b, 1980a,b, 1981; Iqbal and Ochs, 1982; Klee et al, 1980; Means and Dedman, 1980). Our recent observations on microtubules purified by 2 or 3 cycles of warming and cooling procedure indicated the presence of Ca-ATPase which was activated in the presence of calmodulin at a concentration of $\mathrm{Ca}^{2+}$ in the range of $10 \mathrm{M}$ (Ochs and Iqbal, 1980). The Ca-sensitive ATPase associated with microtubular proteins which Ihara et al (1979) found to be stimulated by the addition of tubulin dimer, is likely to be calmodulin-dependent, as calmodulin has been found to be associated with the tubulin dimer (Kumagai and Nishida, 1979). The demonstration of Ca-ATPase activity in microtubules does not, at the present time, indicate whether the observed enzyme activity is due to the presence of enzyme itself as a component of microtubule associated proteins or if the enzyme is an artifact coprecipitating during the purification procedure. Our results also suggest that during the purification procedure a significant amount of calmodulin coprecipitates with microtubular proteins, as was observed by Kumagai and Nishida (1979). The presence of calmodulin in microtubular preparations was indicated by the reduction in the activity of microtubule associated Ca-ATPase when TFP was incorporated in the assay system.

The exact regulatory process involving calmodulin is under extensive investigation. It is possible that the level of calmodulin itself is regulated in the tissue. $125 \mathrm{I}$ labelled $\mathrm{CDR}$ has been found to bind at least four different proteins (LaPorte and Storm, 1978), including proteins of molecular weight 38,000 daltons isolated from retina and of 70,000 daltons isolated from bovine brain (Sharma et al, 1978). Recently, membranes isolated from guinea pig brain have also been found to bind calmodulin (Vandermeers et al, 1978). In regards to the onset of axoplasmic transport process in growing animals, it is not known whether the level of calmodulin changes with growth and, if there is any change it bears any functional relationship with the transport in nerve fibers. Changes in various proteins and enzymes have been observed to show a correlation with the ontogenesis of electrical activity in CNS of developing chick embryos (Iqbal and Talwar, 1970, 1971; Iqbal and Sharma, 1980; Talwar and Iqbal, 1972; Zaheer et al, 1968).

The mechanism of fast axoplasmic transport is not clearly understood. One currently held view of fast transport involves a 'rachet mechanism', somewhat similar to muscle contraction. The microtubules and/or neurofilaments provide the rails and the various components transported are considered to be bound to transport filaments which are moved along the microtubules and/or

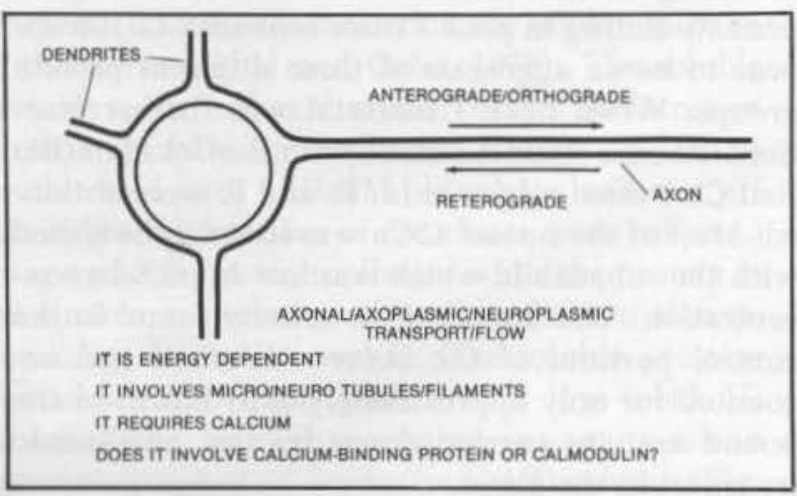

Figure 5. METABOLIC ROLES OF CALMODULIN. 
neurofilaments by means of cross-bridges. The energy required for the transport is provided by the hydrolysis of ATP by a Ca-ATPase found present in the nerve (Khan and Ochs, 1974), probably located on the cross-bridges (Ochs and Iqbal, 1980). Calmodulin could be conceived to play an active role in this process. Our results have shown that the calmodulin isolated from nerve activates nerve $\mathrm{Ca}$ ATPase under normal physiological concentrations of Ca (Iqbal, Garg and Ochs, 1979). In addition to the modulation of Ca-ATPase, calmodulin could be involved in the regulation of $\mathrm{Ca}$ concentrations as well as in the process of assembly-disassembly of microtubules (Marcuma et al, 1978) in the cell. A fast transport of a small amount of calmodulin (Iqbal and Ochs, 1978a, 1979a, 1980a,e, 1982; Erickson et al, 1980) or of a calmodulin-like protein (Tashiro et al, 1980) raises the possibility that calmodulin could be carried down as an integral part of the transport filament and modulating the
Ca-ATPase found associated with microtubules, thus providing energy by the hydrolysis of ATP (Ochs, 1972). Our recent experiments have shown that the exposure of nerve to TFP which binds to calmodulin, causes a block in the transport of proteins (Iqbal and Ochs, 1979a,b). Whether the block of transport is due to an interaction between TFP and calmodulin and thus preventing the modulatory effects of calmodulin, such as the activity of Ca-ATPase, is under investigation.

Acknowledgements, the work was supported by grants fron the NIH, NSF, and in part the American Diabetes Association, Indiana Affiliate. Thanks are due to Professor S. Ochs for providing laboratory facilities, to Roger McIntosh and Lance McKitrick for technical assistance and to Miss Marsha Hunt for typing the manuscript.

(Note: References available on request). 